\title{
Multilayer hexamethyldisiloxane film as bottom antireflective coating for ArF lithography
}

\author{
L. A. Wang ${ }^{\text {a) }}$ and H. L. Chen \\ Institute of Electro-Optical Engineering, National Taiwan University, Taipei, Taiwan, Republic of China
}

(Received 2 June 1999; accepted 17 September 1999)

\begin{abstract}
A new multilayer bottom antireflective coating (BARC) layer composed of hexamethyldisiloxane (HMDSO) film stack is demonstrated for ArF lithography. The required optical constant of each layer can be tuned by varying the gas flow rate ratio of oxygen to HMDSO in an electron cyclotron resonance plasma enhanced chemical vapor deposition process, and the deposition can be completed in one step without changing to other materials. The measured swing effect is shown significantly reduced by adding such a multilayer BARC on both $\mathrm{Al} / \mathrm{Si}$ and silicon crystal substrates. The capability of applying the multilayer BARC to various highly reflective substrates is discussed. Additionally, the multilayer BARC is shown to be capable of providing larger thickness-controlled tolerances than a single-layer BARC. (C) 1999 American Vacuum Society.
\end{abstract}

[S0734-211X(99)18806-1]

\section{INTRODUCTION}

According to the recent Semiconductor Industry Association roadmap, the ArF excimer laser based lithography is regarded as the main technology that would lead to the generation of $130 \mathrm{~nm} .{ }^{1}$ Since the critical dimension control caused by highly reflective substrates becomes stricter in the $193 \mathrm{~nm}(\mathrm{ArF})$ wavelength than in the previous longer ones, a high performance antireflective coating layer for various highly reflective substrates is therefore desirable in $193 \mathrm{~nm}^{2}$ In the integrated circuit process it is generally hard to find an appropriate spin-coated organic material that can match various highly reflective substrates. Conversely, an inorganic bottom antireflective coating (BARC), which is made for example by a conventional chemical vapor deposition (CVD) ${ }^{3}$ may provide the possibility of completely eliminating the reflectance for various highly reflective substrates due to the tunability of both composition and thickness. However, the material composition and thickness of a single-layer inorganic BARC is found hard to be well controlled to match various reflective substrates. A concept of using a gradient absorption multilayer BARC has been reported, ${ }^{4}$ and materials such as silicon oxynitride have been studied ${ }^{5}$ for various reflective substrates to improve the thickness controlled tolerance.

Here we demonstrate a novel multilayer BARC for ArF lithography. The BARC is composed of an hexamethyldisiloxane (HMDSO) film stack deposited by the conventional electron cyclotron resonance-plasma enhanced chemical vapor deposition (ECR-PECVD) process. Note that HMDSO is liquid, relatively cheap, and easily processed as compared to the gases used in the conventional deposition. ${ }^{6-8}$ HMDSO films of different composition and optical properties working in $193 \mathrm{~nm}$ can be obtained by varying the gas flow rate ratio of oxygen HMDSO. The swing effects of resist coated on both $\mathrm{Al} / \mathrm{Si}$ and silicon crystal substrates before and after adding such a multilayer BARC are to be compared. We also

${ }^{a)}$ Electronic mail: lon@ccms.ntu.edu.tw discuss the practicality of using the HMDSO films as multilayer BARC for other highly reflective substrates such as polysilicon (poly-Si), tungsten silicon $(\mathrm{W}-\mathrm{Si})$, aluminum silicon $(\mathrm{Al}-\mathrm{Si})$, and gate structures. The tolerance analysis of thickness control of using such a multilayer BARC will be discussed.

\section{CONCEPT OF MULTILAYER BARC STRUCTURE}

In a multilayer BARC, the extinction coefficient of each BARC layer is gradually increased layer by layer, and is highest at the bottom as schematically shown in Fig. 1. The combination of the multilayer thin film stack creates a gradual change of optical constants to minimize the reflectance at each BARC layer interface. The light reflected from a highly reflective substrate can be absorbed layer by layer. Thus, the graded-absorption multilayer BARC structure can be used for various reflective substrates. ${ }^{4,5}$

For the convenience of the deposition process, the number of layers and the kinds of materials used in a film stack should be as few as possible. In addition, the thickness of the BARC layer should be as small as possible. This is because the BARC layer needs to be etched away after the resist is developed, and the small thickness will help the resist pattern on the BARC layer remain as intact as possible during the etching process to avoid significant variation in critical dimension.

\section{EXPERIMENT SETUP}

The HMDSO films were deposited by employing an ECR-PECVD facility. The dc bias was fixed at $800 \mathrm{~V}$ during the deposition and the substrate was not heated. The gases used were $\mathrm{HMDSO}, \mathrm{O}_{2}$, and $\mathrm{Ar}$, and the chamber was set at $7 \times 10^{-3}$ Torr during deposition. The reflectance spectra were measured by using an optical spectrometer (Hitachi, U3501). The optical constant of an HMDSO film was obtained by employing the resistance-temperature method. ${ }^{9}$ 


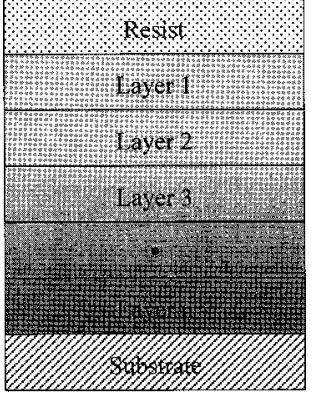

FIG. 1. Schematic diagram of a multilayer BARC.

\section{RESULTS AND DISCUSSION}

\section{A. Optical properties of HMDSO films}

The optical constants of various HMDSO films were measured at $193 \mathrm{~nm}$, and the result is shown in Fig. 2. When the gas flow rate ratios increase, the extinction coefficient $k$ first decreases rapidly and then becomes saturated. Conversely, the refractive index $n$ varies slowly with the ratio. The extinction coefficient has large tuning range from 0.035 to 1.180 as the gas flow rate ratios decrease, which is suitable to be used in a multilayer BARC as we described in Sec. II. In this work, by changing the flow rate ratio of gases $\mathrm{O}_{2}$ to HMDSO, we can complete a multilayer HMDSO BARC in a continual way without changing to other materials.

\section{B. HMDSO film as multilayer BARC structure}

To realize the concept of multilayer BARC structure, the HMDSO films having optical constants (1.632, 0.068), $(1.716,0.517)$, and $(1.691,1.18)$ were chosen as constituents of a three-layer structure. The thickness of each layer was 25 $\mathrm{nm}$. The layer that had the highest absorption was deposited at the bottom, and absorption then decreased layer by layer upwards.

\section{Multilayer BARC structure for a silicon crystal substrate}

The three-layer BARC was added to reduce the reflectance between silicon crystal substrate and resist (Microli-

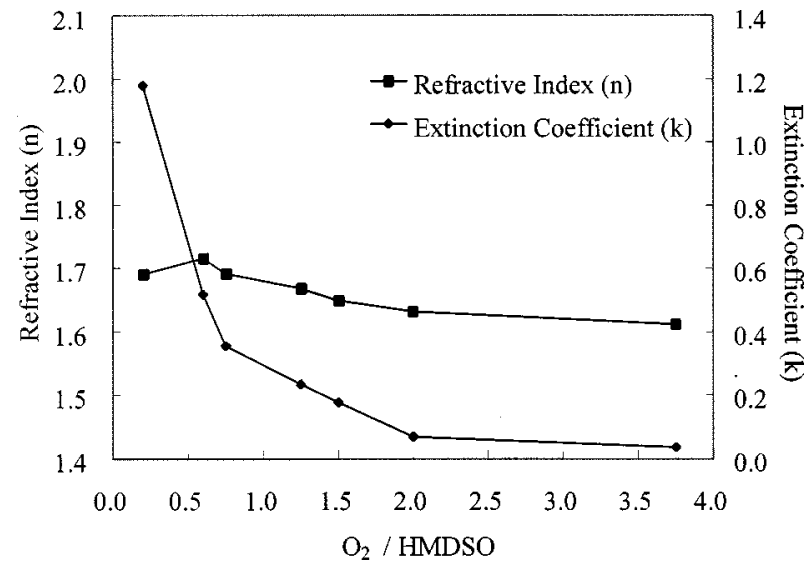

FIG. 2. Optical constant dependence of HMDSO films on gas flow rate ratio.
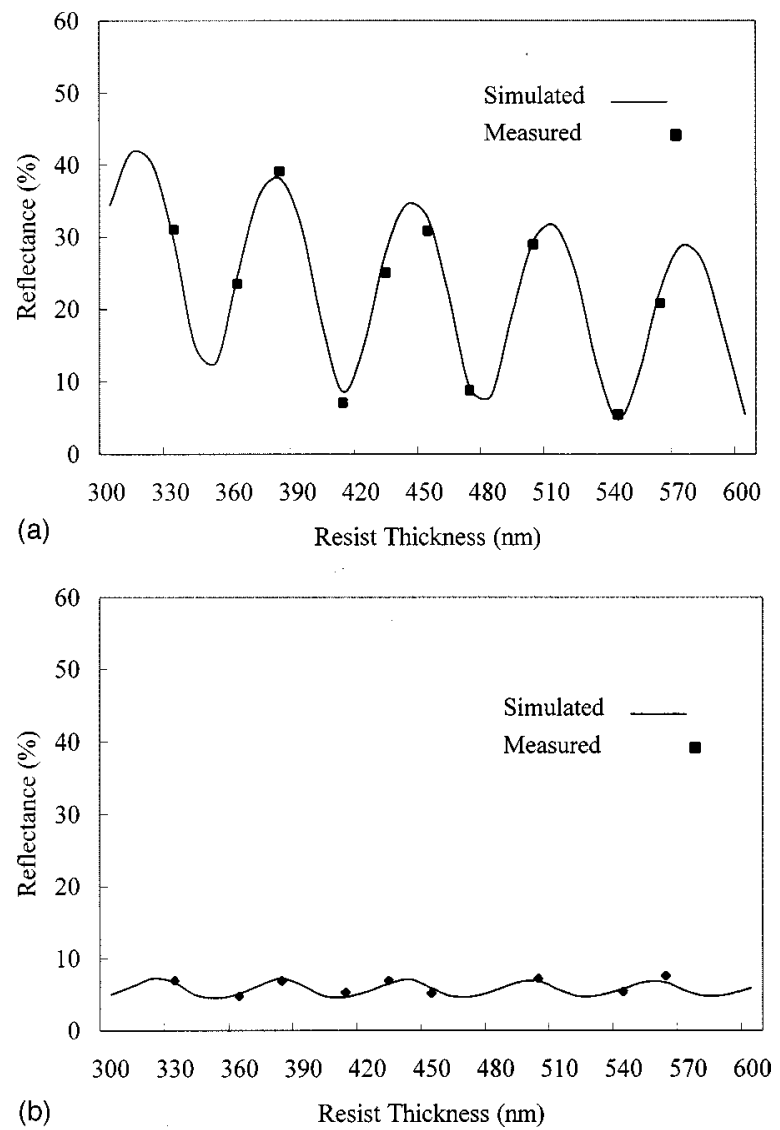

FIG. 3. Reflectance swing curves of TER-1 resist coated on a silicon wafer (a) before and (b) after the deposition of a multilayer BARC.

thography Chemical Corp., TER-1) interfaces. By the thin film theory, ${ }^{10}$ the simulated reflectance can be reduced to $0.41 \%$.

The swing effect caused by optical interference between the fields reflected from both the air/resist and resist/ substrate interfaces will induce the control issue of critical dimension in an optical lithography process. The reflectance swing curves of TER-1 resist coated on a silicon wafer with and without adding BARC layer were simulated and measured, and the results are shown in Figs. 3(a) and 3(b), respectively. The reflectance exhibits a sinusoidal variation from about $5 \%$ to $40 \%$ for the resist thickness ranging from 300 to $600 \mathrm{~nm}$ before adding the BARC layer, and the variation is reduced to about $4 \%-8 \%$ after the addition. The results indicate that the use of such an HMDSO based multilayer BARC can significantly reduce the swing effect incurred in the resist, and is then expected to have the capability of improving on critical dimension control.

\section{Multilayer BARC structure for an Al film}

Similarly, the same HMDSO three-layer structure was added to reduce the reflectance between the resist and aluminum interfaces, all deposited on a silicon crystal substrate. The reflectance can be reduced to $0.58 \%$ theoretically.

The reflectance swing curves of TER-1 resist coated on an aluminum film on a silicon wafer were simulated and mea- 

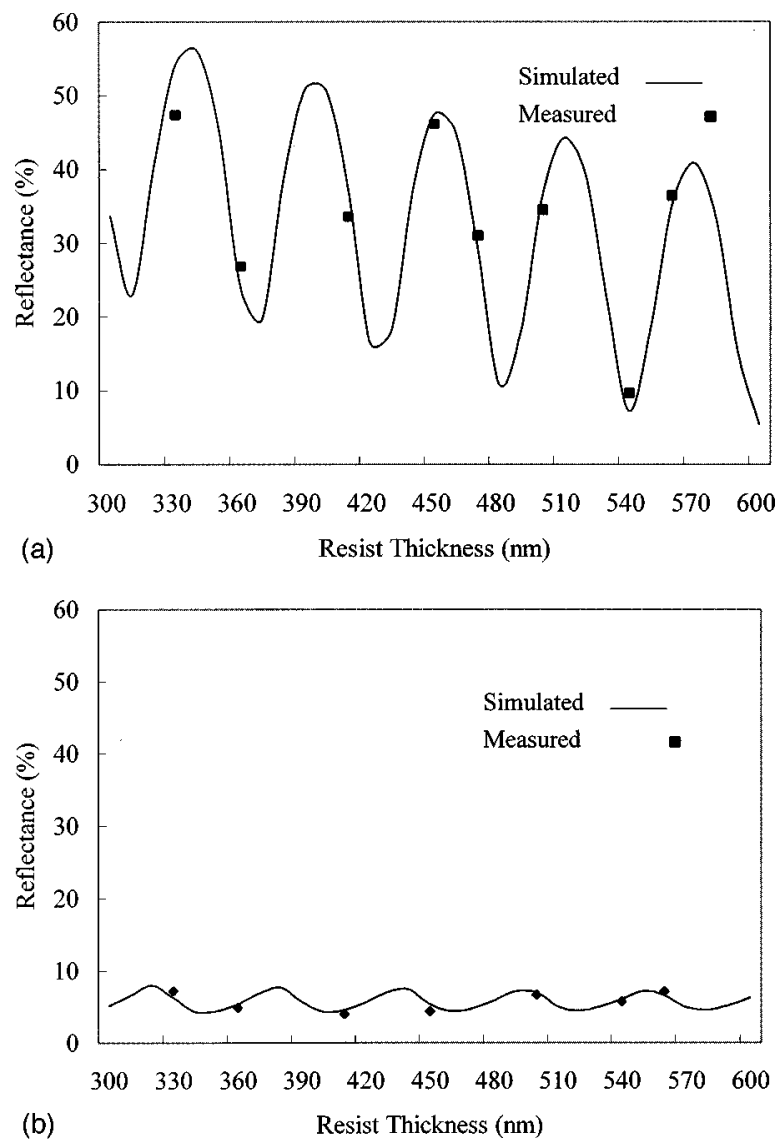

FIG. 4. Reflectance swing curves of the TER-1 resist coated on an Al film (a) before and (b) after deposition of a multilayer BARC.

sured before and after adding the BARC layer as shown in Figs. 4(a) and 4(b), respectively. The reflectance originally exhibits a sinusoidal variation from about $5 \%$ to $60 \%$ and is reduced to about $4 \%-8 \%$ when the BARC layer is applied for the same range of resist thickness. The results show that the same BARC layer can also significantly reduce the swing effect incurred in resist coated on an aluminum film.

\section{Multilayer BARC structure for other highly reflective substrates}

The practicality of using such an HMDSO based multilayer structure for other highly reflective substrates such as poly- $\mathrm{Si}, \mathrm{W}-\mathrm{Si}$, and $\mathrm{Al}-\mathrm{Si}$ is evaluated by simulating their optical performance, and the results are summarized in Table I. The optical constants of these highly reflective materials at $193 \mathrm{~nm}$ are obtained from Ref. 11. Results show this BARC would reduce reflectance at the interface of resist/ high reflective materials to less than $1 \%$. The HMDSO based multilayer BARC structure is therefore expected to have great potential for these highly reflective materials.

\section{Multilayer BARC structure for a gate structure}

In the gate structure, resist and BARC layers are coated on $\mathrm{SiO}_{2} / \mathrm{W}-\mathrm{Si}$ or $\mathrm{SiO}_{2} / \mathrm{Al}-\mathrm{Si}$ substrate. ${ }^{3}$ However, the thickness variation of the $\mathrm{SiO}_{2}$ layer will cause the reflectance variation from the resist and BARC layer interfaces.
TABLE I. HMDSO-based BARC layers for highly reflective substrates.

\begin{tabular}{|c|c|c|c|}
\hline Substrates & Poly-Si & $\mathrm{W}-\mathrm{Si}$ & $\mathrm{Al}-\mathrm{Si}$ \\
\hline Optical constant at $193 \mathrm{~nm}^{\mathrm{a}}$ & $(0.90,2.30)$ & $(1.15,2.24)$ & $(0.15,1.15)$ \\
\hline Reflectance from resist/substrate & $51.2 \%$ & $42.8 \%$ & $82.4 \%$ \\
\hline $\begin{array}{l}\text { Reflectance from resist/BARC } \\
\text { layer/substrates after adding a } \\
\text { multi BARC layer }\end{array}$ & $0.41 \%$ & $0.37 \%$ & $0.64 \%$ \\
\hline
\end{tabular}

${ }^{\mathrm{a}}$ See Ref. 11.

Figure 5(a) shows for the addition of a single-layer HMDSObased BARC, where the reflectance varies sinusoidally from about $0 \%$ to $24 \%$ with the thickness of the $\mathrm{SiO}_{2}$ layer deposited on a W-Si substrate. However, when an HMDSO-based three-layer BARC is applied, the reflectance variation can be reduced to less than $1 \%$ over $200 \mathrm{~nm} \mathrm{SiO}_{2}$ thickness variation as shown in Fig. 5(b). It indicates that the multilayer BARC can significantly reduce the reflectance variation caused by the thickness variation of the $\mathrm{SiO}_{2}$ layer in a typical gate structure.

\section{Thickness tolerance analysis of BARC layer}

For an HMDSO-based single BARC layer structure with optical constant $(1.716,0.517)$ and thickness of $30.8 \mathrm{~nm}$, the reflectance between resist and BARC layer interfaces can be reduced to $0.20 \%$. The thickness tolerance analysis is shown in Table II(a) which indicates that the reflectance increases to $2 \%-5 \%$ as the thickness tolerance is larger than $30 \%$. Simi-
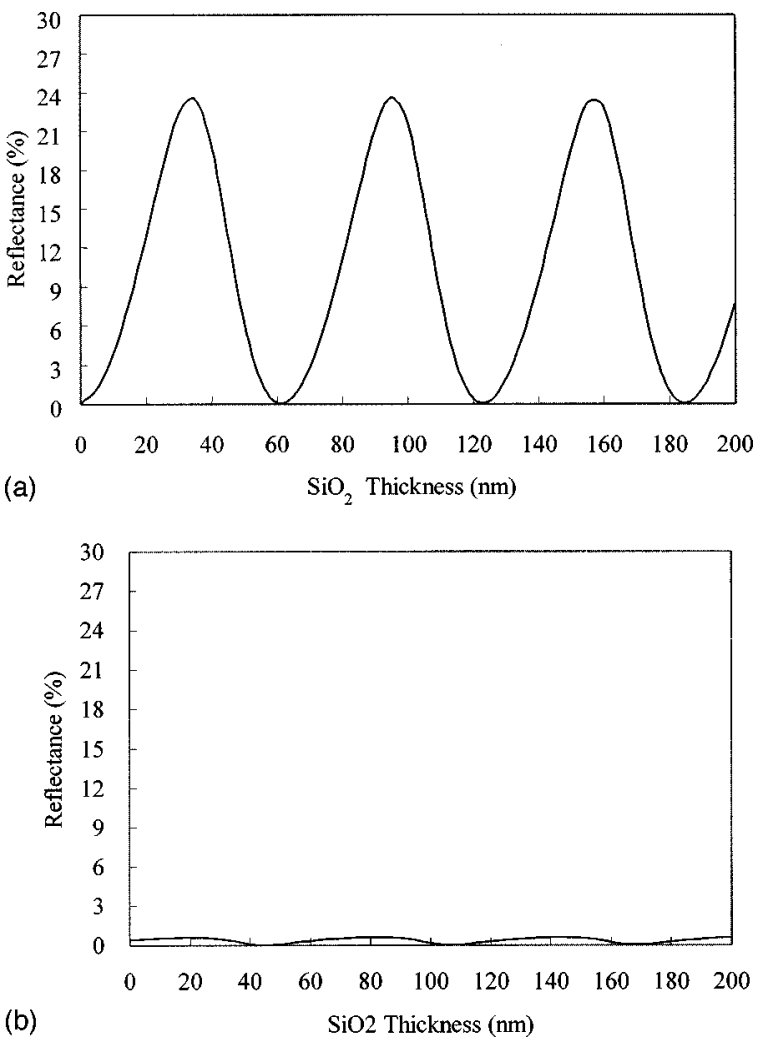

FIG. 5. Dependence of reflectance variation on $\mathrm{SiO}_{2}$ thickness when (a) single-layer, and (b) multilayer BARCs are employed. 
TABLE II. Thickness tolerance analysis of: (a) single layer and (b) multilayer BARCs.

\begin{tabular}{|c|c|c|c|}
\hline $\begin{array}{l}\text { Thickness } \\
\text { tolerance }(\%)\end{array}$ & Reflectance $(\%)$ & $\begin{array}{c}\text { Thickness } \\
\text { tolerance }(\%)\end{array}$ & Reflectance $(\%)$ \\
\hline \multicolumn{4}{|l|}{ (a) Single layer } \\
\hline 0 & 0.20 & +10 & 0.45 \\
\hline-10 & 0.70 & +20 & 1.21 \\
\hline-20 & 2.22 & +30 & 2.24 \\
\hline-30 & 5.00 & +40 & 3.35 \\
\hline-40 & 9.28 & +50 & 4.35 \\
\hline-50 & 15.18 & +60 & 5.10 \\
\hline \multicolumn{4}{|l|}{ (b) Multilayer } \\
\hline 0 & 0.41 & +10 & 0.37 \\
\hline-10 & 0.43 & +20 & 0.35 \\
\hline-20 & 0.44 & +30 & 0.36 \\
\hline-30 & 0.54 & +40 & 0.42 \\
\hline-40 & 1.08 & +50 & 0.51 \\
\hline-50 & 2.71 & +60 & 0.66 \\
\hline
\end{tabular}

larly, Table II(b) shows the thickness tolerance analysis for an HMDSO-based three-layer structure with each layer having $25 \mathrm{~nm}$ thickness. For the same 30\% thickness tolerance, the reflectance is shown to remain less than $1 \%$, indicating that the multilayer structure can significantly increase thickness-controlled tolerance.

\section{CONCLUSION}

The HMDSO film deposited by the ECR-PECVD process is found to be appropriate as a new multilayer BARC struc- ture for ArF lithography. The optical characteristics of HMDSO films can be controlled by varying the oxygen/ HMDSO gas flow rate ratio. The multilayer structure can be deposited in one continual CVD coating process without changing to other materials. The measured reflectance swing effect on both $\mathrm{Al} / \mathrm{Si}$ and silicon crystal substrates is shown to be significantly reduced by adding the HMDSO-based multilayer BARC structure. The same multilayer BARC structure is also shown to have great potential to be used on the other highly reflected substrate such as poly-Si, W-Si, $\mathrm{Al}-\mathrm{Si}$, and gate structure. As compared to a single-layer $\mathrm{BARC}$, the multilayer BARC is shown to be able to provide larger thickness-controlled tolerances.

${ }^{1}$ P. Gargini, J. Glaze, and O. Williams, Solid State Technol. 41, 73 (1998). ${ }^{2}$ Y. Tani, H. Mito, Y. Okuda, Y. Todokoro, T. Tatsuta, M. Sawai, and O. Tsuji, Jpn. J. Appl. Phys., Part 1 32, 5909 (1993).

${ }^{3}$ T. Gocho, T. Ogawa, M. Muroyama, and J. Sato, Jpn. J. Appl. Phys., Part 1 33, 486 (1994).

${ }^{4}$ T. Tanaka, N. Asai, and S. Uchino, Proc. SPIE 2276, 573 (1996).

${ }^{5}$ R. A. Cirelli, G. R. Weber, A. Kornblit, R. M. Baker, F. P. Klemens, and J. Demarco, J. Vac. Sci. Technol. B 14, 4229 (1996).

${ }^{6}$ D. Korzec, D. Theirich, F. Werner, K. Traub, and J. Engemann, Surf. Coat. Technol. 74, 67 (1995).

${ }^{7}$ R. Rochotzki, M. Arzt, F. Blaschta, E. Krebig, and U. H. Poll, Thin Solid Films 234, 463 (1993).

${ }^{8}$ S. Matsuo and M. Kiuchi, Jpn. J. Appl. Phys., Part 2 22, L210 (1983).

${ }^{9}$ T. C. Paulick, Appl. Opt. 25, 562 (1986).

${ }^{10}$ H. A. Macleod, Thin Film Optical Filters (Macmillan, New York, 1986).

${ }^{11}$ T. Ogawa, A. Sekiguchi, and N. Yoshizawa, Jpn. J. Appl. Phys., Part 1 35, 6360 (1996). 